\title{
Nature as a network of morphological infocomputational processes for cognitive agents
}

\author{
Gordana Dodig-Crnkovic ${ }^{\mathrm{a}}$ \\ Chalmers University of Technology, 41296 Gothenburg, Sweden
}

Received 16 November 2016 / Received in final form 20 November 2016 Published online 12 January 2017

\begin{abstract}
This paper presents a view of nature as a network of infocomputational agents organized in a dynamical hierarchy of levels. It provides a framework for unification of currently disparate understandings of natural, formal, technical, behavioral and social phenomena based on information as a structure, differences in one system that cause the differences in another system, and computation as its dynamics, i.e. physical process of morphological change in the informational structure. We address some of the frequent misunderstandings regarding the natural/morphological computational models and their relationships to physical systems, especially cognitive systems such as living beings. Natural morphological infocomputation as a conceptual framework necessitates generalization of models of computation beyond the traditional Turing machine model presenting symbol manipulation, and requires agent-based concurrent resource-sensitive models of computation in order to be able to cover the whole range of phenomena from physics to cognition. The central role of agency, particularly material vs. cognitive agency is highlighted.
\end{abstract}

\section{The necessity of an agent/observer/actor for generation of knowledge}

Information is a concept known for its multiplicity of use and ambiguity in both common, everyday application and in its specific formal definitions throughout different fields of research. However, most people are unaware that matter/energy today is also a concept with uncertain content. Matter that for Democritus was substance of the whole universe, appears today to constitute only $4 \%$ of its observed content [1]. The rest constituting $96 \%$ is labeled "dark matter" (conjectured to explain gravitational effects otherwise unaccounted for) and "dark energy" (introduced to account for the expansion of the universe). We do not know what "dark matter" and "dark energy" actually are. This indicates that our present understanding of the structure of the physical world might need re-examination. By connecting two ambiguous and currently much discussed ideas - information and matter/energy - can we hope to

${ }^{a}$ e-mail: dodig@chalmers.se 
gain more clarity and some new insights about the structures and dynamics of the universe? More and more researchers believe so. One of the approaches proposes formulating physics in terms of information. The idea goes back to Wheeler's "it from bit" [2], the proposal that for us, as cognizing agents, information is the fabric of reality. Our reality results from the processing of information of multimodal signals coming through our senses in combination with information processes in our bodies and brains that unfold on several levels of organisation (scale).

According to Bateson "In fact, what we mean by information - the elementary unit of information - is a difference which makes a difference, and it is able to make a difference because the neural pathways along which it travels and is continually transformed are themselves provided with energy. The pathways are ready to be triggered." [3] p. 460 It is the difference in the world that makes the difference for an agent.

"Information expresses the fact that a system is in a certain configuration that is correlated to the configuration of another system. Any physical system may contain information about another physical system." argues Hewitt [4] p. 293. Combining the two, we end up with the following: Information constitutes a structure consisting of differences in one system that cause the differences in another system. In other words, information is observer relative. In recent years informational reality is the subject of a steady stream of books. Among prominent examples are [5-10]. We define computation as information processing [11] (dynamics of information), using variation of Hewitt's Actor model of concurrent distributed computation [12] in which "computational devices" are conceived as computational agents - informational structures capable of acting on their own behalf. Actors are the universal primitives of concurrent distributed digital computation. In response to a message that it receives, an Actor can make local < decisions>, create more Actors, send more messages, and designate how to respond to the next message received. Computation is "conceived as distributed in space, where computational devices communicate asynchronously and the entire computation is not in any well-defined state." (An Actor can have information about other Actors that it has received in a message about what it was like when the message was sent.) Turing model of computation is a special case of the Actor model [13]. Unlike other models of computation that are based on mathematical logic, set theory, algebra, etc., the Actor model is based on physics, especially quantum physics and relativistic physics.

It is physical process of intrinsic morphological change in the informational structure, physical implementation of information, as there is no information without physical implementation [14]. Both information and computation appear on many different levels of organisation/abstraction/resolution/granularity of matter/energy in space-time. In this article we present a framework constructed to provide a unified infocomputational natural philosophy connecting domain-specific knowledge from variety of sciences, from physics to cognitive science.

\section{Relational infocomputation as reality for an agent}

Matter and energy are higher order concepts derived from interactions of observers with the universe, including mutual interactions among observers. This does not mean that the reality is just an agreed upon imagination. A world exists on its own with human observers/agents in. We discover its structures and processes, patterns and laws through interactions via self-structuring of information as a result of morphological computation [15-17]. The most rigorous model of scientific knowledge production models both the world and the agent researching the world. The reality for a cognitive agent is built upon the external information available and agent's own 
information processing architecture. The reality of an amoeba or a robot differs from the reality of a human $[18,19]$. We take our reality as default, but from robotics and studies of other living beings we learn about the diversity of possible "realities" for different kinds of agents. The "reality" for other organisms might look like quite differently, depending on sensors and actuators that an organism possesses [19]. Philosophers, among others Kant [20] and Block [21] addressed the question of the difference between the world in itself, with its full richness and that which cognizing agents can observe. We as humans can observe with our senses, even when augmented by experiments and reasoning, only a part of that which exists. The same insight comes from roboticists who learn the role of the embodiment for the ability to adequately perceive, act and persist in the world, through the interactions and the use of resources from the environment [22]. Reality for an agent is essentially dependent on the type of agency of the system that explores it. The agent could be bacteria, animal, plant or human who sees "the reality", "the world". For more on bacterial cognition see [23-25]. That which exists for an agent is a result of infocomputational interactions with the environment and shared within societies of communicating agents. Minsky [26] describes how human mind builds on interactions of simple mindless agents he terms the societies of mind. The process of "negotiating reality" in networks neurons as information processing agents has commonalities with information processing going on in bacterial swarms and colonies.

In this article we present the current state of the art of understanding of the connection between different layers of information processing in nature, that starts with physics, and continues via chemistry, biology to cognitive science and distributed concurrent information processing in societies of cognitive agents. Informational approaches go one step deeper than our present day level of understanding of nature, building a layer beneath the structures of the currently used matter/energyin-space/time paradigm connecting agents information processing with the world as information on the interface [27]. This does not mean that current sciences are thereby invalidated with their ideal of objectivity (that is inter-subjectivity). Observer-dependent does not mean subjective. Taking into account a well-defined observer (agent) in the model of reality, makes explicit which in classical physics was implicit. As Goyal [28] explains, an observer in classical physics was a highly idealized omnipotent agent looking at the world from nowhere. In the words of Fields [29], a "Galilean observer" is a bare "point of view", able to observe and conceptualize the world without affecting it or being affected. What infocomputational framework makes possible is the inclusion of a model of an observer into the model of the universe (for that kind of observer).

\section{Morphological infocomputation in cognitive systems}

Through billions of years of evolution, nature has developed information processing networks in living organisms capable of coping with complexity of their environments in an effective way: acting, learning, anticipating. The step from inanimate to animate matter is still largely unknown, but there are indications that natural processes of self-assembly/self-organization of increasingly complex structures, from elementary particles, to atoms, molecules and their aggregates have spontaneously led to first "molecular machines" and then to first self-organized cells that in the next step formed first multicellular organisms. With each step in the evolution, new and more complex communication forms have emerged. While atoms typically interact/communicate by exchanging electrons, molecules exchange electrons, atoms or other (smaller) molecules. Only recently we learned about the essential role of viruses and bacteria for the development of the life on earth. Agents exchanged among 
organisms can be bacteria, viruses, or pieces of DNA that can actively alter the organism that receives them, as they continue to act within an organism. All those processes of communication along with self-organization (autopoiesis) unfold in living systems that are thermodynamically open, as they need energy in order to persist, in the regime on the edge of chaos [30,31].

All living beings have an ability to adapt to the environment, and learn so to increase their own chances of survival. Maturana and Varela were the first to propose that the life itself can be understood as a process of cognition [32,33]. Building on Maturana and Varela's understanding of cognition the author proposed an infocomputational constructive framework $[18,34-37]$ with the aim to explain how increasingly complex structures develop as a result of information processing in nature. These information processes can be modeled as natural morphological computation, in the network of networks of physical processes where constant exchange (communication) of data (signals) among agents establishes new structures on physical, chemical and biological levels. Instead of symbol-manipulation, morphological computation is a result of physical objects interactions [38]. Natural computation [39] can be used to explain the emergent phenomena by complexification of information through processes of signals exchange at different levels of organization. The new framework is used as a tool for studying cognitive systems such as living organisms or artifactual cognitive agents on different levels of complexity. Denning argues that computing should be seen as a natural science [40].

In order to adequately model cognition, a model of computation is generalized from the abstract symbol manipulation of the Turing machine type to the information processing in physical systems. This solves the problem of early computationalist approaches to cognition, based on the Turing machine model of computation, such as symbol grounding problem, lack of embodiment and resource agnosticism. In the new infocomputational approach, information is created and structured on different levels or scales in cognitive systems, and its dynamics corresponds to different kinds of morphological (physical, intrinsic) computation [38,41].

Complex systems in nature have already inspired a number of computational models, including artificial neural networks, genetic algorithms and deep learning algorithms, and development continues in the direction of cognitive computing that can be found in living organisms. It is instructive to compare the new approach with the existing models of computation - open versus closed systems, generative versus predetermined sequences, parallel versus sequential processes, and so on. In [42], we investigate the consequences of the generalization of the concept of computation with application to morphological processes in which the morphology of a cognitive system determines the dynamics of its informational structure.

An organism presents an information exchange network evolved and developed according to a few basic principles, using sensors and actuators, with Hebbian and Bayesian learning, that constantly builds and adapts its own structure in interaction with the environment. Of special interest are processes of sensory-to-motor transformation of data through self-organization of information as morphological computation; memory and learning by changes of morphology, and decision-making by signal vs. symbol processing, based on morphological computations. This applies to different classes of cognitive agents - from the simplest single-celled organisms and their networks to the most complex agents such as humans.

Recasting physical, chemical, biological and cognitive processes into the common framework of morphological computation provides a unified approach to the evolution of matter and life in infocomputational networks of agents communicating with given "languages" corresponding to their level of organisation - from elementary particles, atoms, molecules, cells, organisms, societies and ecologies. According to computing nature/natural computationalism [41] every physical system is computational, but 
there are many different sorts of computations going on in nature seen as a network of agents/actors exchanging "messages". The simplest agents communicate with simplest messages such as elementary particles (with 12 kinds of matter and 12 kinds of anti-matter particles) exchanging 12 kinds of force-communicating particles. An example from physics that we can recast into the actor model is Yukawa's theory of strong nuclear force modeled as exchange of mesons (as messages), which explained the interaction between nucleons. Complex agents/actors like humans communicate through languages that use very complex messages for communication. Also, exchange of information causes change of actors. Those changes are simple in a simple actor such as an elementary particle, which can change its state (quantum numbers), while in complex agents with memory, communication often results in substantial changes in the agents' own architecture and thus in its way of response.

Natural computational systems as networks of agents exchanging messages are in general asynchronous concurrent systems. Conceptually, agent-based models and actor models are closely related, and as mentioned, understanding of interactions between agents in interaction networks fits well in those frameworks.

\section{It from $(\mathrm{Qu})$ bit through quantum computation}

As fundamental science, physics describes the basic reality of the world for a human agent, obtained by learning process of scientific research. It addresses the totality of physical existence, the universe, as it is perceived, experienced, conceptualized, theorized, shared and used by human agents. By physical we mean all inter-subjectively established phenomena - objects and processes - that are possible to detect either directly by our senses or via instruments and theoretical tools. Physics can be formulated as science of information, as argued by Goyal [28], Vedral [43], Fields [29], Zenil [44] and Wharton [45]. Goyal examines the idea of "it from bit" from the point of view of quantum theory, in order to clarify why and how information acquires a central role when moving from classical physics to quantum physics, and how the informational point of view is allowing us to deeper understand the nature of quantum reality, through the use of quantum theory to implement informational protocols, and through formulation of information-inspired principles to derive predictions of quantum theory. The question of what comes first, physics or information, is addressed by Vedral who argues that both physics and information science profit from interaction with each other. According to Zenil of central importance is research into information and computation applied to biology, which until now was notoriously difficult to model, and successfully resisted the famous "unreasonable effectiveness of mathematics in the natural sciences" proclaimed by Wigner [46]. Biology as a basis of cognition is important in closing the loop between an observer and the world observed.

When studying the physical character of information, it is good to remember that the physical has a variety of meanings in the sense of Wittgenstein's family resemblance. According to Collier as quoted in [47] "Quine takes the physical to be anything accessible to the senses or inferences thereof. Ladyman, Ross, Collier and Spurrett [48] take the physical to be the most fundamental laws of our (part of) the universe. Information is physical in both of these senses. It can be related to the causal, which always has physical parameters."

On the quantum mechanical level of physical reality there are qubits, "So, what does that leave us with? - asks Deutsch and offers an answer [49]." Not "something for nothing": Information does not create the world ex nihilo; nor a world whose laws are really just fiction, so that physics is just a form of literary criticism. But a world in which the stuff we call information, and the processes we call computations, really do have a special status." This idea gets further developed in his Constructor Theory of 
Information [50]. The same emphasis on the dual nature of information/computation is found in Dodig Crnkovic [51] as well as Zenil [52].

\section{Nature as hierarchical self-structured infocomputational network}

Present day computers are very different from the early stand-alone calculating machines that Turing helped construct, designed to mechanize computation of mathematical functions. Computers today are networked and largely used for worldwide communication and a variety of information processing and knowledge management. They are cognitive tools of extended mind, in the sense of Clark and Chalmers [53], used in social interactions and they provide ever-growing repositories of information. Moreover, contemporary computers play an important role in the control of physical processes and thus connect directly to the physical world in automation, and control. Apart from classical engineering and hard-scientific domains, computing has in recent decades pervaded a vast variety of new fields including biology and social sciences, humanities and arts - all previously considered as typical soft, non-mechanical and non-automatable domains.

Computational processes running in networks of networks (such as the internet) can be modeled as distributed, reactive, agent-based and concurrent computation. The main criterion of success of this computation is not its termination, but its behavior - response to changes, its speed, generality and flexibility, adaptability, and tolerance to noise, error, faults, and damage. Internet, as well as operating systems and many database management systems are designed to operate continuously and termination would mean an error. As a generalization of the traditional algorithmic Turing machine model of computation, in which the computer was an isolated box provided with a suitable algorithm and an input, left alone to compute until the algorithm terminated, both technological and natural computation are based on interaction, i.e. communication of computing processes with each other and with the environment. In natural systems, computation is information processing that can proceed on both symbolic and sub-symbolic (signal-processing) levels. For human cognitive processes it means that not only the execution of an algorithm can be seen as computation, but also learning, reasoning, processing of information from sense organs, etc.

Hewitt [13] characterizes the Turing machine model as an internal (individual) framework and the Actor model of concurrent computation as an external (sociolog$i c a l$ ) model of computing. This tension between an (isolated) individual one and (interacting) social many can be found in Cottam et al. [54] who distinguish "conceptual umbrella of entity and its ecosystem" and Schroeder's view that "Information can be defined in terms of the categorical opposition of one and many, leading to two manifestations of information, selective and structural. These manifestations of information are dual in the sense that one always is associated with the other." Here information is directly related with computation defined as information processing [54].

The frequent objection against the computational view of the universe, elaborated by Zenil, is: if the universe computes, what are the input and the output of its computation? This presupposes that a computing system must have an input from the outside and that it must deliver some output to the outside world. But for the actor system [13] input for the next state in computation is its preceding state. Within the framework of computing nature, the whole universe computes its own next state from its current state [41]. As all of physics is based on quantum mechanical layer of information processing, zero-point (vacuum) oscillations can be seen as constant input for the computational network of the universe. What causes different processes in the universe is the interaction or exchange of information between its parts. The universe 
is a result of evolution from the moment of big bang or some other primordial state, through the complexification of the relationships between its actors by computation as a process of self-organisation of its informational structure. Physical forces are established through particle exchanges (message exchanges), which necessarily connect particles into a web of physical interactions as manifestation of natural laws. The whole of the universe is in the state of permanent flow, far from steady state, which results in forming increasingly complex structures [55].

The objection upheld by [56] that not all of the universe can be computational, as it is too powerful a metaphor, can be answered by pointing out the layered architecture of the computing nature, as not all of computation is the same - computation is proceeding on many scales, on many levels of hierarchical organization. Moreover, in tandem with computation, universe is described by information, representing its structures. Given that computation follows physical laws, or represents/implements physical laws, a generative model of the universe can be devised such that some initial network of informational processes develops in time into increasingly complex (fractal, according to Kurakin [55]) information structures. The parallel could be drawn between natural computation and atomic theory of matter, which is another general theory that implies that all of matter is made of atoms (and void).

Unified theories are common and valued in physics and other sciences, and computing nature is such a unified framework. It is therefore not unexpected that physicists are found among the leading advocates of the unified theory of informational and computational universe - from Wheeler [57], via Feynman [58], to Fredkin [59], Lloyd [6], Wolfram [60], Goyal [28] and Chiribella [61]. For the views of the latter two physicists on the topic of the informational universe, see the special issue of the journal Information titled Information and Energy/Matter [47], the special issue of the journal Entropy titled Selected Papers from Symposium on Natural/Unconventional Computing and its Philosophical Significance [62] and the special issue of the journal Information titled "Physics of Information" [63].

\section{Self-organized complexity and Bayesian/Jaynesian observers}

Apart from the direct answers to the question of the physical nature of information [14] and the informational nature of physics [28], the computing nature approach introduces a new understanding of the variety of topics including the role of an observer in the self-organized complexity, mechanisms of chemical affinities relevant for the origins of life, to the issues of representation and logic used to relate an agent with its real world (Umwelt) [19]. An observer or rather an agent undergoing interaction with the world, can be a cognizing subject as a researcher constructing theory, or a simple physical system like a chemical molecule interacting with its surroundings. Yoshitake and Saruwatari [64] describe the interaction of an agent with the world as an act of measurement. They address articulation of information from the physical world via interactions alone, generalizing the extensive measurement theory. Matsuno and Salthe [65] consider material agency for naturalizing contextual meaning in case of "observers" (agents) that are molecules involved in interactions used for studying chemical affinity. An important natural example of this material agency is suggested as responsible for the origins and evolution of life. Living agents possess increasingly complex structures and dynamics that evolved through the interactions with the environment. We already mentioned studies of bacteria swarms as complex cognitive systems [23-25]. Organisms with nervous systems present an important qualitative change in the information processing organisms, as nervous system enables self-representation, making it possible for an organism to use symbols in order to remember or anticipate - enabling considerable speed up in processing and 
increased expressiveness of representation. As the next step in the development come organisms with the brain with the function of integration of multimodal information. In recent years Bayesian theories have gained considerable success in modeling information processes of the brain, and have been applied to he study of perception, learning, reasoning and decision-making. They have also become popular in neuroscience, and varieties of potential neurobiological mechanisms explaining Bayesian brain have been proposed.

"Bayesian theories view the brain as an observer (or a collection of observers) that integrates information with the goal of accurately predicting the state of the world (which corresponds to maximizing information or minimizing uncertainty." "The brain is not a unified observer but a multitude of neuronal observers, and it is to be expected that what is logical given the information of one local observer may appear illogical from the perspective of another observer having different information. If physical systems follow logic, then we can use probabilities to describe information that is local in space and time. The present work provides a first and necessary step towards a description of what one physical entity knows about another." [66]

Jaynes developed information-based, epistemic, inductive probability using the maximum entropy inference (or logic) to convert prior information for an observer into prior probability distribution. While Jaynes applied probability theory to the problems of inference facing scientists (notably in statistical mechanics), Phillips [67] and Fiorillo [68] independently consider the implications of Jaynes's work for our understanding of how the brain can perform inference. Phillips studies how complexity can be adaptively self-organized by using probabilistic context-sensitive inference. Jaynes's probabilistic inference is seen not only as the logic of science, but generally as the logic of life. Fiorillo argues for Jaynes's approach, in which probabilities are always conditional on a state of knowledge of an agent through the rules of logic, as expressed in the maximum entropy principle which provides the objective means for deriving probabilities, as well as a unified account of information and logic (knowledge and reason). Fiorillo's article suggests how to identify a probability distribution over states of one physical system (an "object") conditional only on the biophysical state of another physical system (an "observer"). Even though the aim is to show "what it means to perform inference and how the biophysics of the brain could achieve this goal", this approach exemplifies the idea of relational information as defined by Hewitt [13].

\section{History of the idea of the computing universe}

Konrad Zuse was the first to suggest (in 1967) that the physical behavior of the entire universe is being computed on a basic level, possibly on cellular automata, by the universe itself which he referred to as "Rechnender Raum" or Computing Space/Cosmos [69]. Consequently, Zuse was the first pancomputationalist (naturalist computationalist). Here is how Chaitin explains pancomputationalism:

"And how about the entire universe, can it be considered to be a computer? $Y e s$, it certainly can, it is constantly computing its future state from its current state, it's constantly computing its own time-evolution! And as I believe Tom Toffoli pointed out, actual computers like your PC just hitch a ride on this universal computation!" [70]

Fredkin in his Digital Philosophy [71] suggests that particle physics can emerge from cellular automata. For "computational universe" according to Fredkin, "reality is a software program run on a cosmic computer." The universe is digital, time and space discrete. Humans are software running on a universal computer. Even Wolfram in his $A$ New Kind of Science advocates a pancomputational view, a new dynamic 
kind of reductionism, in which complexity of behaviors and structures found in nature are derived (generated) from a few basic computational mechanisms. Natural phenomena are the products of computation processes. In a computational universe new and unpredictable phenomena emerge as a result of simple algorithms operating on simple computing elements such as e.g. cellular automata, and complexity originates from bottom-up driven emergent processes. Cellular automata are equivalent to a universal Turing machine. Wolfram's critics remark however that cellular automata do not evolve beyond a certain level of complexity. The mechanisms involved do not produce evolutionary development. Actual physical mechanisms at work in the physical universe appear to be quite different from simple cellular automata. Wolfram meets this criticism by pointing out that cellular automata are models and as such surprisingly successful ones.

\section{Natural/physical/morphological computation}

Natural computation [39] deals with computation in the physical world, which has brought a fundamentally new understanding of computation $[42,72]$. Research in natural computation is characterized by a bidirectional learning [73] as the natural sciences are rapidly absorbing ideas of information processing, while computing assimilates ideas, concepts and approaches from natural sciences. Natural computation is a study of computational systems that include: computing techniques that take inspiration from nature for the development of novel problem-solving methods; use of computers to simulate natural phenomena; and computing with natural materials (e.g., molecules, atoms). Fields of research within natural computing are among others Biological Computing/Organic Computing, Artificial Neural Networks, Swarm Intelligence, Artificial Immune Systems, computing on continuous data, Membrane Computing, Artificial Life, DNA computing, Quantum computing, Neural computation, Evolutionary computation, evolvable hardware, self-organizing systems, emergent behaviors, machine perception and Systems Biology. Evolution is a good example of the natural computational process. The kind of computation it performs is Morphological computation $[15,74]$. The result of this computation is the body shape and material optimized for the class of organisms in a given type of environment.

According to computing nature/natural computationalism/pancomputationalism $[18,41,75,76]$ one can view the time development (dynamics) of physical states in nature as information processing, and learn about its computational characteristics. Such processes include self-assembly, developmental processes, gene regulation networks, gene assembly in unicellular organisms, protein-protein interaction networks, biological transport networks, and similar.

Natural computing has specific criteria for the success of a computation. While for the Turing model of computation, the halting problem is a central issue; instead the adequacy of the computational behaviour is central for natural computing. An organic computing system e.g. adapts dynamically to the current conditions of its environments by self-organization, self-configuration, self-optimization, self-healing, self-protection and context-awareness. In many areas, we have to computationally model emergence not being algorithmic [77,78] which makes it interesting to investigate computational characteristics of non-algorithmic natural computation (subsymbolic, analog). Much like the research in other disciplines of Computing such as AI, SE, and Robotics, Natural computing is an interdisciplinary research field, and has a synthetic approach, unifying knowledge from a variety of related fields. Research questions, theories, methods and approaches are used from Computer Science (such as Theory of automata and formal languages, Interactive computing), Information Science (e.g. Shannon's theory of communication), ICT studies, mathematics (such 
as randomness, Algorithmic theory of information), Logic (e.g. pluralist logic, game logic), Epistemology (especially naturalized epistemologies), evolution and Cognitive Science (mechanisms of information processing in living organisms) in order to investigate foundational and conceptual issues of Natural computation and information processing in nature.

"(O) ur task is nothing less than to discover a new, broader, notion of computation, and to understand the world around us in terms of information processing." [73] This development necessitates what [79] calls computational research "beyond the constraints of normal science". In other words, a paradigm shift or [80] "the second generation models of computation", such as Petri nets and Process Algebra, the first generation being the Turing machine model. Abramsky summarizes the process of changing paradigm of computing as follows:

"Traditionally, the dynamics of computing systems, their unfolding behavior in space and time has been a mere means to the end of computing the function which specifies the algorithmic problem which the system is solving. In much of contemporary computing, the situation is reversed: the purpose of the computing system is to exhibit certain behaviour. (...) We need a theory of the dynamics of informatic processes, of interaction, and information flow, as a basis for answering such fundamental questions as: What is computed? What is a process? What are the analogues to Turing completeness and universality when we are concerned with processes and their behaviours, rather than the functions which they compute?" [80]

The first generation models of computation originated from problems of formalization of mathematics and logic. In the second generation models of computation, previous isolated systems with limited interactions with the environment are replaced by processes or agents for which the interactions with each other and with the environment form are fundamental. As a result of interactions among agents and with the environment, complex behaviors emerge. The basic building block of this interactive approach is an agent, and the fundamental operation is interaction. This approach works at both macro-scale (processes in operating systems, software agents on the Internet, transactions, etc.) and on micro-scale (program implementation, down to hardware). This view of the relationship between information and computation presented in [80] agrees with the ideas of infocomputational naturalism which is based on the same understanding of an agent-based interactive model of natural computation and its relation to (agent-based) information. In his article: What is computation? Concurrency versus Turing's Model, Hewitt [13] makes the following analysis of the relationship between Turing machines and concurrent computing processes:

"Concurrency is of crucial importance to the science and engineering of computation in part because of the rise of the Internet and many-core architectures. However, concurrency extends computation beyond the conceptual framework of Church, Gandy [1980], Gödel, Herbrand, Kleene [1987], Post, Rosser, Sieg [2008], Turing, etc. because there are effective computations that cannot be performed by Turing Machines. In the Actor model [Hewitt, Bishop and Steiger 1973; Hewitt 2010], computation is conceived as distributed in space where computational devices communicate asynchronously and the entire computation is not in any well-defined state. (An Actor can have information about other Actors that it has received in a message about what it was like when the message was sent.) Turing's Model is a special case of the Actor Model." [13]

\section{Complexity and emergence at hierarchy of levels of organisation}

If computation is understood as a physical process, if Nature computes with physical bodies as objects while physical laws are governing the process of computation, 
then the computation necessarily appears on many different levels of organization in nature. Natural sciences provide such a layered view of Nature. One sort of computation processes will be found on the quantum-mechanical levels of elementary particles, atoms and molecules; yet another on the level of classical physics. On the organizational level of biology, different processes (computation, information processing) are going on in biological cells, tissues, organs, organisms, and eco-systems. Social interactions are governed by still another kind of communicative/interactive processes. In short, computation on a given level of organization is implementation of the laws that govern the interactions between different constituent parts. Consequently, what happens on every next level of organization is that a set of rules governing the system switches to the new level [81]. Recently, simulation tools are being developed which allow for the study of the behavior of complex systems modeled computationally. For the analysis of the time development of dynamic systems various simulation techniques exist, from purely mathematical approaches, e.g. equation based modeling, simulated by iterative evaluation, to formal modeling approaches, such as Petri Nets and Process Algebra together with Object-oriented and Agent-oriented simulation methods based on the emulation of constituent system elements.

In short, solutions are being sought in natural systems with evolutionary developed strategies for handling complexity in order to improve modeling and construction of complex networks of massively parallel autonomous computational systems. The research in the theoretical foundations of Natural computing is needed to improve our understanding on the fundamental level of computation as information processing which underlies all of the computing in nature.

\section{Summary}

This paper presents a framework for a unified understanding of cognitive systems through self-structuring processes of morphological infocomputation on the levels of physics, chemistry and biology, through embodied information and morphological computation. We

- argue for infocomputationalism as a new philosophy of nature, providing a basis for unification of currently disparate understanding of natural, formal, technical, social and behavioral phenomena;

- offer computational interpretation of information dynamics in nature;

- provide explanation of an observer-dependent reality, that is reality for an agent as a result of cognition built upon relational infocomputational processes;

- elucidate most frequent misunderstandings regarding the models of computation and their relationships to physical systems, especially cognitive ones such as living beings;

- suggest the necessity of generalization of the models of computation beyond the traditional Turing machine model and acceptance of "second generation" models of computation in order to be able to cover the whole range of phenomena from physics to cognition.

The developments supporting infocomputational naturalism are found in among others Complexity Theory, Systems Theory, Theory of Computation (Natural Computing, Organic Computing, Unconventional Computing), Cognitive Science, Neuroscience, Information Physics, Agent Based Models of social systems and Information Sciences, Robotics (especially Developmental Robotics) as well as Bioinformatics and Artificial Life [41]. 
The author is grateful to anonymous reviewers for their useful comments. This research is supported by the Swedish Research Council project MORCOM@COGS, Morphological Computing in Cognitive Systems.

\section{References}

1. NASA, Dark Energy, Dark Matter, 2016

2. J.A. Wheeler, Information, Physics, Quantum: The Search for Links, in Complexity, Entropy, and the Physics of Information, ed. W. Zurek (Addison-Wesley, Redwood City, 1990)

3. G. Bateson, Steps to an Ecology of Mind: Collected Essays in Anthropology, Psychiatry, Evolution, and Epistemology, ed. P. Adriaans, J. van Benthem (University of Chicago Press, Amsterdam, 1972)

4. C. Hewitt, What Is Commitment? Physical, Organizational, and Social, in Coordination, Organizations, Institutions, and Norms in Agent Systems II, ed. P. Noriega, J. VazquezSalceda, G. Boella, O. Boissier, V. Dign (Springer-Verlag, Berlin, Heidelberg, 2007), p. 293

5. H. von Baeyer, Information: The New Language of Science (Harvard University Press, Cambridge Mass., 2004)

6. S. Lloyd, Programming the Universe: A Quantum Computer Scientist Takes on the Cosmos (Knopf, New York, 2006)

7. C. Seife, Decoding the Universe: How The New Science of Information is Explaining Everything in the Cosmos, from Our Brains to Black Holes (Viking, New York, 2006)

8. V. Vedral, Decoding Reality: The Universe as Quantum Information (Oxford University Press, Oxford, 2010)

9. P. Davies, N.H. Gregersen, Information and the Nature of Reality from Physics to Metaphysics (Cambridge University Press, 2010)

10. M. Burgin, Theory of Information: Fundamentality, Diversity and Unification (World Scientific Pub Co., Singapore, 2010)

11. M. Burgin, Super-Recursive Algorithms (Springer-Verlag, New York, 2005)

12. C. Hewitt, P. Bishop, P. Steiger, A Universal Modular ACTOR Formalism for Artificial Intelligence, in IJCAI - Proceedings of the 3rd International Joint Conference on Artificial Intelligence, ed. N.J. Nilsson (William Kaufmann, Standford, 1973), p. 235

13. C. Hewitt, What is computation? Actor Model versus Turing's Model, in A Computable Universe, Understanding Computation \& Exploring Nature As Computation, ed. H. Zenil (Imperial College Press, 2012)

14. R. Landauer, Information is Physical, Phys. Today 44, 23 (1991)

15. R. Pfeifer, F. Iida, Morphological computation: Connecting body, brain and environment, Japanese Sci. Mon. 58, 48 (2005)

16. R. Pfeifer, G. Gomez, Morphological computation - connecting brain, body, and environment, in Creating Brain-like Intelligence: From Basic Principles to Complex Intelligent Systems, ed. K.B. Sendhoff, O. Sporns, E. Körner, H. Ritter, K. Doya (Springer, Berlin, 2009), p. 66

17. G. Dodig-Crnkovic, The info-computational nature of morphological computing, in Philosophy and Theory of Artificial Intelligence Volume 5, ed. V.C. Müller (Springer, Berlin, 2013), p. 59

18. G. Dodig-Crnkovic, Information, Computation, Cognition. Agency-Based Hierarchies of Levels, Fundamental Issues of Artificial Intelligence (Springer International Publishing, Switzerland, 2016), Vol. 376, pp. 141-159

19. J. von Uexküll, A Stroll through the Worlds of Animals and Men, in Instinctive Behavior ed. C. Schiller (International Universities Press, New York, 1957), p. 5

20. S. Palmquist, Kant's System of Perspectives (University Press of America, Lanham, 1993) 
21. N. Block, Consciousness, Accessibility and the mesh between psychology and neuroscience, Behav. Brain Sci. 30, 481 (2007)

22. R. Pfeifer, J. Bongard, How the Body Shapes the Way We Think - A New View of Intelligence (MIT Press, 2006)

23. E. Ben-Jacob, Social behavior of bacteria: from physics to complex organization, Eur. Phys. J. B 65, 315 (2008)

24. E. Ben-Jacob, Bacterial Self-Organization: Co-Enhancement of Complexification and Adaptability in a Dynamic Environment, Phil. Trans. R. Soc. Lond. A 361, $1283(2003)$

25. S. Schauder, B.L. Bassler, The languages of bacteria, Genes. Dev. 15, 1468 (2001)

26. M. Minsky, The Society of Mind (Simon and Schuster, New York, 1986)

27. O. Rössler, Endophysics: The World as an Interface (World Scientific, Singapore, New Jersey, London, Hong Kong, 1998)

28. P. Goyal, Information Physics - Towards a New Conception of Physical Reality, Information 3, 567 (2012)

29. C. Fields, If physics is an information science, what is an observer? Information 3, 92 (2012)

30. I. Prigogine, I. Stengers, Order out of Chaos: Man's new dialogue with nature (Flamingo, 1984)

31. I. Prigogine, From Being to Becoming: Time and Complexity in the Physical Sciences (W.H. Freeman, San Francisco, CA, 1980)

32. H. Maturana, F. Varela, Autopoiesis and cognition: The realization of the living (D. Reidel Pub. Co., Dordrecht, Holland, 1980)

33. J. Stewart, Cognition = life: Implications for higher-level cognition, Behav. Process. 35, $311(1996)$

34. G. Dodig-Crnkovic, Significance of Models of Computation, from Turing Model to Natural Computation, Minds Mach. 21, 301 (2011)

35. G. Dodig-Crnkovic, Info-Computational Philosophy Of Nature: An Informational Universe With Computational Dynamics, in Festschrift for Søren Brier ed. C. P. and T.T. Sørensen Bent (CBS University Press, 2011), p. 97

36. G. Dodig-Crnkovic, Modeling Life as Cognitive Info-Computation, in Computability in Europe 2014. LNCS, ed. A. Beckmann, E. Csuhaj-Varjú, K. Meer (Springer, Berlin, Heidelberg, 2014), p. 153

37. G. Dodig-Crnkovic, Why we need info-computational constructivism, Constr. Found. 9, $246(2014)$

38. G. Dodig-Crnkovic, Info-computationalism and morphological computing of informational structure, in Integral Biomathics, ed. P.L. Simeonov, L.S. Smith, A.C. Ehresmann (Springer, Berlin, Heidelberg, 2012), p. 97

39. G. Rozenberg, T. Bäck, J.N. Kok, Handbook of Natural Computing (Springer, Berlin, Heidelberg, 2012)

40. P. Denning, Computing is a natural science, Commun. ACM 50, 13 (2007)

41. G. Dodig-Crnkovic, V. Müller, A Dialogue Concerning Two World Systems: InfoComputational vs. Mechanistic, in Information and Computation, ed. G. Dodig Crnkovic, M. Burgin (World Scientific Pub. Co. Inc., Singapore, 2011), p. 149

42. M. Burgin, G. Dodig-Crnkovic, A Taxonomy of Computation and Information Architecture, in Proceedings of the 2015 European Conference on Software Architecture Workshops (ECSAW'15), ed. M. Galster (ACM Press, New York, 2015)

43. V. Vedral, Information and Physics, Information 3, 219 (2012)

44. H. Zenil, Information Theory and Computational Thermodynamics: Lessons for Biology from Physics, Information 3, 739 (2012)

45. K. Wharton, Quantum States as Ordinary Information, Information 5, 190 (2014)

46. E. Wigner, The Unreasonable Effectiveness of Mathematics in the Natural Sciences, Commun., Pure Appl. Math. 13 (1960)

47. G. Dodig-Crnkovic, Information and Energy/Matter, Information 3, 751 (2012) 
48. J. Ladyman, D. Ross, D. Spurrett, J. Collier, Everything Must Go: Metaphysics Naturalised (Clarendon Press, Oxford, 2007)

49. D. Deutsch, Quantum theory, the Church-Turing Principle and the universal quantum computer, Proc. R. Soc. Lond. A 400, 97 (1985)

50. D. Deutsch, C. Marletto, Constructor Theory of Information, Proc. R. Soc. A 471, 1 (2015)

51. G. Dodig-Crnkovic, Physical Computation as Dynamics of Form that Glues Everything Together, Information 3, 204 (2012)

52. H. Zenil, A Computable Universe. Understanding Computation 66 Exploring Nature as Computation, ed. H. Zenil (World Scientific Publishing Company/Imperial College Press, Singapore, 2012)

53. A. Clark, D.J. Chalmers, The Extended Mind, Analysis 58, 7 (1998)

54. G. Dodig-Crnkovic, R. Giovagnoli, Computing Nature (Springer, Heidelberg, 2013)

55. A. Kurakin, The self-organizing fractal theory as a universal discovery method: The phenomenon of life, Theor. Biol. Med. Model. 8, 1 (2011)

56. G. Piccinini, Computation in Physical Systems, Stanford Encycl. Philos. (2012)

57. J.A. Wheeler, It from Bit, in At Home in the Universe (Am. Inst. Phys., 1994), p. 295

58. R.P. Feynman, Simulating Physics with Computers, Int. J. Theor. Phys. 21, 467 (1982)

59. E. Fredkin, Finite Nature, in XXVIIth Rencontre de Moriond (1992)

60. S. Wolfram, A New Kind of Science (Wolfram Media, 2002)

61. G. Chiribella, G.M. D'Ariano, P. Perinotti, Quantum Theory, Namely the Pure and Reversible Theory of Information, Entropy 14, 1877 (2012)

62. G. Dodig-Crnkovic, R. Giovagnoli, Natural/Unconventional Computing and Its Philosophical Significance, Entropy 14, 2408 (2012)

63. H. Zenil, G. Dodig-Crnkovic, Special Issue "Physics of Information", Information 5 (2014)

64. M. Yoshitake, Y. Saruwatari, Extensional Information Articulation from the Universe, Information 3, 644 (2012)

65. K. Matsuno, S. Salthe, Chemical Affinity as Material Agency for Naturalizing Contextual Meaning, Information 3, 21 (2011)

66. S.L. Kim, C.D. Fiorillo, Describing realistic states of knowledge with exact probabilities, in AIP Conference Proceedings 175\%. 060008 (2016)

67. W.A. Phillips, Self-Organized Complexity and Coherent Infomax from the Viewpoint of Jaynes's Probability Theory, Information 3, 1 (2012)

68. C.D. Fiorillo, Beyond Bayes: On the Need for a Unified and Jaynesian Definition of Probability and Information within Neuroscience, Information 3, 175 (2012)

69. K. Zuse, Calculating Space. Translation of "Rechnender Raum" (MIT Technical Translation, 1970)

70. G. Chaitin, Epistemology as Information Theory: From Leibniz to $\Omega$, in Computation, Information, Cognition - The Nexus and The Liminal, ed. G. Dodig Crnkovic (Cambridge Scholars Pub., Newcastle, UK, 2007), p. 2

71. E. Fredkin, Digital Mechanics: An Information Process Based on Reversible Universal Cellular Automata, Physica D 45, 254 (1990)

72. G. Dodig-Crnkovic, Significance of Models of Computation from Turing Model to Natural Computation, Minds Mach. 21, 301 (2011)

73. G. Rozenberg, L. Kari, The many facets of natural computing, Commun. ACM 51, 72 (2008)

74. R. Pfeifer, F. Iida, G. Gomez, Morphological Computation for Adaptive Behavior and Cognition, Int. Congr. Ser. 1291, 22 (2006)

75. G. Dodig-Crnkovic, Investigations into Information Semantics and Ethics of Computing (Mälardalen University Press, Västerås, Sweden, 2006)

76. G. Dodig-Crnkovic, Where Do New Ideas Come From? How Do They Emerge? Epistemology as Computation (Information Processing), ed. C. Calude (World Scientific, 2007), p. 1 
77. S.B. Cooper, B. Löwe, A. Sorbi, New Computational Paradigms. Changing Conceptions of What is Computable, Springer Mathematics of Computing Series, XIII, ed. S.B. Cooper, B. Löwe, A. Sorbi (Springer, 2008)

78. A. Sloman, Beyond Turing Equivalence, in Machines and Thought: The Legacy of Alan Turing (vol I), ed. A. Clark, P.J.R. Millican, (OUP, The Clarendon Press, 1996), p. 179

79. J. Collier, Hierarchical dynamical information system with a focus on biology, Entropy 5, $102(2003)$

80. S. Abramsky, Information, Processes and Games, in Philosophy of Information, ed. J. van Benthem, P. Adriaans (North Holland, Amsterdam, The Netherlands, 2008), p. 483

81. F. Heylighen, Evolutionary Transitions: How do levels of complexity emerge? Complexity 6, $53(2000)$

Open Access This is an Open Access article distributed under the terms of the Creative Commons Attribution License (http://creativecommons.org/licenses/by/4.0), which permits unrestricted use, distribution, and reproduction in any medium, provided the original work is properly cited. 